\title{
Approved for Human Use Product
}

National Cancer Institute

\section{Source}

National Cancer Institute. Approved for Human Use Product. NCI Thesaurus. Code C121840.

A product has been approved for use in humans. 